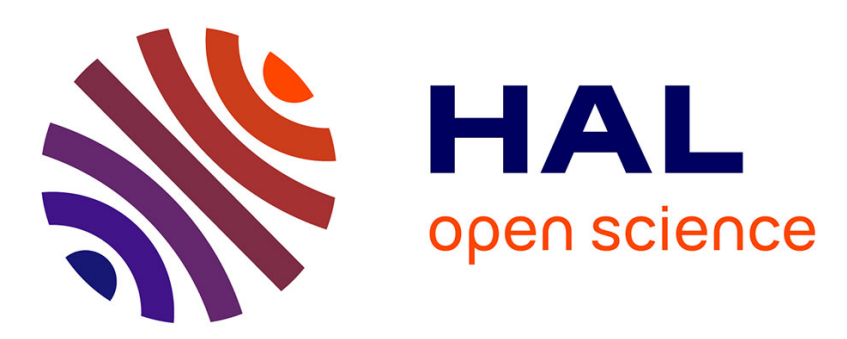

\title{
La mesure de flux intégrés de neutrons rapides au moyen de compteurs de Geiger-Muller à cathodes activables
}

Pierre Bayle, Henri Rakotondrafara, Michel Levy, Annie Vallée

\section{To cite this version:}

Pierre Bayle, Henri Rakotondrafara, Michel Levy, Annie Vallée. La mesure de flux intégrés de neutrons rapides au moyen de compteurs de Geiger-Muller à cathodes activables. Revue de Physique Appliquée, 1966, 1 (2), pp.97-100. 10.1051/rphysap:019660010209700 . jpa-00242705

\section{HAL Id: jpa-00242705 https://hal.science/jpa-00242705}

Submitted on 1 Jan 1966

HAL is a multi-disciplinary open access archive for the deposit and dissemination of scientific research documents, whether they are published or not. The documents may come from teaching and research institutions in France or abroad, or from public or private research centers.
L'archive ouverte pluridisciplinaire HAL, est destinée au dépôt et à la diffusion de documents scientifiques de niveau recherche, publiés ou non, émanant des établissements d'enseignement et de recherche français ou étrangers, des laboratoires publics ou privés. 


\title{
LA MESURE DE FLUX INTÉGRÉS DE NEUTRONS RAPIDES AU MOYEN DE COMPTEURS DE GEIGER-MULLER A CATHODES ACTIVABLES
}

\author{
par Pierre BAYLe et Henri RAKotondrafara, \\ Centre de Physique Nucléaire, Faculté des Sciences, Toulouse. \\ Michel LEVY et Mule Annie VALLÉE, \\ Laboratoire Central de l'Armement, Arcueil, Seine.
}

\begin{abstract}
Résumé. - Pour mesurer des flux intégrés de neutrons rapides de $14 \mathrm{MeV}$ on emploie des compteurs de Geiger-Müller à cathodes activables d'aluminium, d'argent, de cuivre, de laiton, ou d'acier inoxydable. La mesure du taux de comptage du détecteur après activation est directement liée au flux total de neutrons. Le flux minimal mesurable est défini par le bruit de fond du compteur, la valeur maximale mesurable par son temps mort. La dose minimale détectable est de $5 \mathrm{~m}$ rad avec des cathodes de cuivre, la dose maximale est de 40 rad, dans le cas de flux faibles et de compteurs en acier inoxydable.
\end{abstract}

\begin{abstract}
Geiger-Müller counters with activable cathodes of aluminium, silver, copper, brass or stainless steel are used for the measurement of integral fluxes of $14 \mathrm{MeV}$ fast neutrons.

The measurement of the detector count rate after activation is directly related to the neutrons total flux. The minimum flux measurable is determined by the counter background, the maximum value measurable by its dead time.

The minimum detectable dose is $5 \mathrm{~m}$ rad with copper cathodes, the maximum dose is $40 \mathrm{rad}$, for stainless steel cathode counters and low fluxes.
\end{abstract}

Introduction. - L'activation de certains isotopes par des neutrons rapides offre un moyen de mesurer leurs flux et d'obtenir une dosimétrie approchée ; les détecteurs à seuil, en particulier, on fait l'objet d'un grand nombre de recherches [1] à [8]. Le procédé le plus souvent employé consiste à mesurer l'activité de l'échantillon, après irradiations, en le plaçant devant un compteur de Geiger-Müller à fenêtre. Les problèmes d'angle solide de détection, de rétrodiffusion et de rayonnements secondaires compliquent la méthode et rendent difficile l'interprétation des résultats. L'intégration du matériau activable au compteur lui-même permet d'éliminer ces inconvénients.

Peirson [9] a utilisé, pour la détection des neutrons thermiques, un compteur dont la cathode est constituée par une feuille d'indium supportée par une feuille de cuivre. L'ensemble est placé à l'intérieur d'une enveloppe en verre. Le flux minimal détectable est de 0,04 neutrons $\mathrm{cm}^{-2} \mathrm{~s}^{-1}$.

Glover [10] utilise un compteur proportionnel en aluminium, pour la mesure de faibles flux de neutrons rapides, avec un remplissage composé de $90 \%$ d'argon et de $10 \%$ de méthane. Le compteur est capable de détecter un flux minimal de $10^{3} \mathrm{n}$ (rapides) $\mathrm{cm}^{-2} \mathrm{~s}^{-1}$.

Nous basant sur ces deux études, nous avons construit des compteurs de Geiger-Müller dont la cathode puisse être activée par les neutrons de $14 \mathrm{MeV}$. Avec une telle géométrie, l'angle solide de détection des particules $\beta$ venant de l'élément activé est voisin de $2 \pi$.

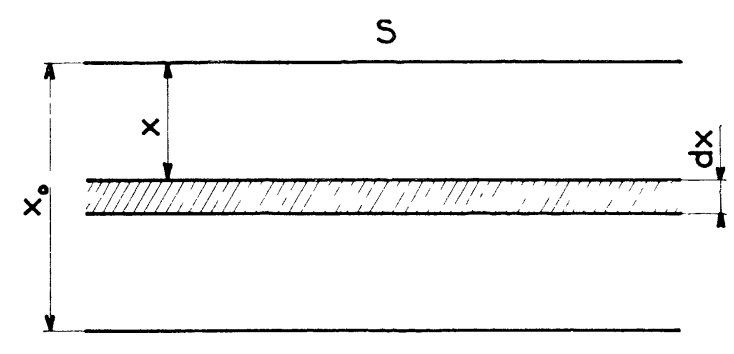

FIG. 1.

Sélection des cathodes utilisables. - Le matériau à employer doit répondre à un certain nombre de critères :

1) Les sections efficaces d'activation pour les neutrons rapides sont beaucoup plus faibles que celles pour les neutrons lents ou thermiques. Le rôle parasite que risquent de jouer les impuretés est alors très atténué et il n'est pas indispensable d'avoir un métal de très haute pureté. Par contre, la section efficace doit avoir une valeur suffisante pour que l'activité ne soit pas trop faible.

2) La période radioactive du radioélément produit doit avoir une valeur comprise entre 5 minutes et 3 heures environ. 
En effet, si la période est trop courte, l'activation est considérable mais la saturation est atteinte rapidement et la limite supérieure des flux intégrés mesurables est faible; une période longue correspond à une faible activation et c'est la sensibilité qui est insuffisante; quelle que soit la cathode employée, le temps d'irradiation doit être inférieure à $2 T$ ( $T$ étant la période du radioélément produit). Comme on le sait en effet, pour un temps d'irradiation inférieur à deux fois la période. l'activation est sensiblement proportionnelle à ce temps d'irradiation.

3) Le travail d'extraction de la cathode doit être aussi élevé que possible, les impulsions parasites étant d'autant moins nombreuses que le travail d'extraction de la cathode est plus élevé [11].

4) Le matériau sélectionné doit pouvoir s'usiner sans trop de difficultés et doit posséder une bonne résistance mécanique.

5) L'épaisseur de la cathode doit être assez grande pour que l'étanchéité soit assurée. Il est doncindispensable de faire une correction d'autoabsorption pour les particules $\beta$ qu'elle produit.

Soit un élément de surface d'épaisseur $\mathrm{d} x$, se trouvant à la profondeur $x$.

L'activité à la surface, due à cet élément est :

$$
A=S \sigma \rho \mathrm{e}^{-\mu x} \mathrm{~d} x
$$

$S$ : étant la surface de la source,

$\sigma$ : son activité spécifique,

$\rho$ : sa densité,

$\mu$ : le coefficient d'absorption linéaire.

L'activité due à l'épaisseur $x_{0}$ totale de la cathode est :

$$
\begin{aligned}
& A=S \sigma \rho \int_{0}^{x_{0}} \mathrm{e}^{-\mu x} \mathrm{~d} x \\
& A=(S \sigma \rho / \mu)\left(1-\mathrm{e}^{-\mu x_{0}}\right) .
\end{aligned}
$$

L'activité totale de la source étant

$$
A_{0}=S \sigma \rho x_{0}
$$

le facteur $f$ de correction est :

$$
f=A / A_{0}=\frac{1}{\mu x_{0}}\left(1-e^{-\mu x_{0}}\right) .
$$

Tous les résultats ci-dessous sont donnés après cette correction.

Toutes les conditions qui précèdent nous ont conduit à sélectionner l'aluminium, le cuivre (ou le laiton), l'argent, l'acier inoxydable.

Détecteurs réalisés ( $f g .2)$. - Notre premier souci a été celui de la simplicité afin que le détecteur soit aussi peu coûteux que possible et puisse être utilisé de façon très courante.

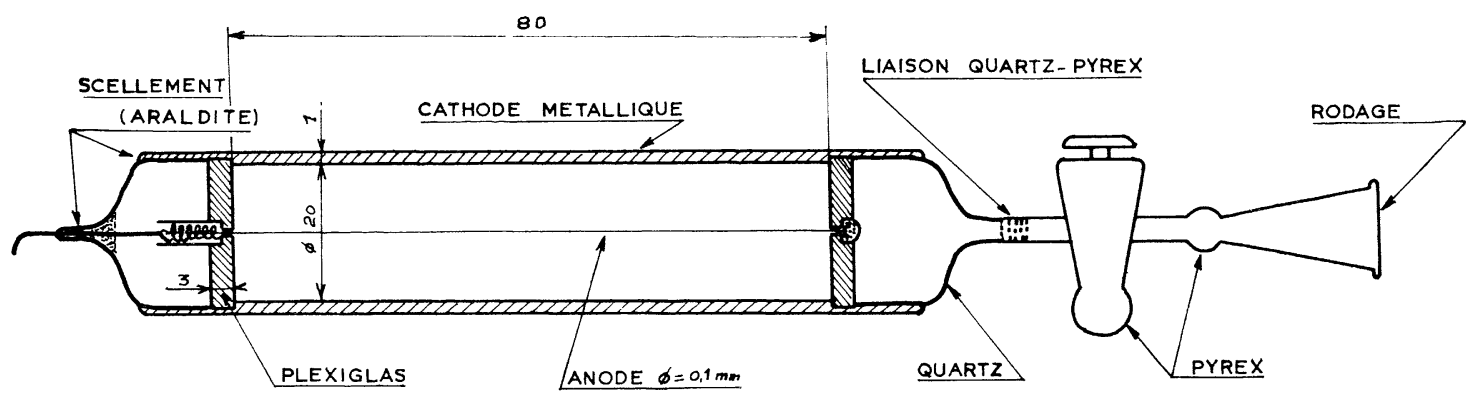

Fig. 2. - Schéma des compteurs réalisés.

La cathode est un cylindre de $20 \mathrm{~mm}$ de diamètre épais de $1,5 \mathrm{~mm}$, terminé par deux épaulements où sont logés des disques de plexiglass qui permettent de centrer exactement l'anode. Celle-ci est un fil de tungstène (diamètre : $0,1 \mathrm{~mm}$ ).

Les matériaux ont été sélectionnés de telle sorte que l'activité mesurée provienne de la cathode en presque totalité. L'araldite et le plexiglass ne sont pratiquement pas activés. Les embouts de silice fondue donnent la réaction ${ }_{14}^{28} \mathrm{Si}(\mathrm{n}, \mathrm{p})$ avec une section efficace $\sigma=250 \mathrm{mb}$ pour des neutrons de $14 \mathrm{MeV}$. Le radioélément ainsi produit, ${ }_{13}^{28} \mathrm{Al}$, a une période de 2,3 minutes. Il suffit d'attendre 10 à 15 minutes après irradiation pour que cette activité ne joue plus qu'un rôle négligeable dans les mesures ; enfin l'activité de l'anode est toujours négligeable. Le remplissage est constitué par de l'argon
(90 torr) et une vapeur organique (10 torr), cyclohexane, acétone, méthylal ou isobutane. Les différentes catégories de compteurs n'ont pas été remplis avec les mêmes mélanges. Pour que les résultats obtenus soient comparables, il faut que les compteurs présentent des performances sinon identiques $d u$ moins voisines. Il n'était donc pas intéressant d'adopter un seul remplissage standard. Nous nous sommes efforcés d'obtenir des compteurs dont les paliers aient des caractéristiques aussi voisines que possible (longueur comprise entre 150 et 200 volts, pente de 2 à $4 \%$ par 100 volts), avec des temps morts très voisins $\left(1,5 \times 10^{-4} \mathrm{~s}\right.$ pour une surtension de 100 volts).

Le circuit électronique associé est classique : les impulsions provenant de l'anode sont mises en forme dans un préamplificateur Philips PW 4071 
suivi d'un amplificateur PW 4072/O1. Elles passent ensuite dans une échelle de comptage.

Conditions d'irradiation. - Les neutrons de $14 \mathrm{MeV}$ sont produits par réaction $\mathrm{D}$. T. dans un générateur électrostatique S. A. M. E. S. de 150 kilovolts. Les flux de neutrons réalisés sont contrôlés de façon permanente par un compteur à scintillations d'anthracène, préalablement étalonné.

Nous avons obtenu des flux de l'ordre de $10^{8}$ neutrons par seconde dans tout l'angle solide. Les détecteurs sont disposés à une distance de l'ordre de $4 \mathrm{~cm} \mathrm{du}$ centre de la cible, l'axe du compteur étant normal avec la direction moyenne des neutrons incidents. Avec une telle disposition géométrique, le flux moyen traversant le compteur est de $5.10^{5}$ neutrons $\mathrm{cm}^{-2} \mathrm{~s}^{-1}$.

Limites des flux mesurables. - Les performances pratiques des compteurs sont limitées par le bruit de fond dans la mesure des faibles activités, et par le temps mort pour les activités intenses.

a) Dose minimale. - Elle est imposée par le bruit de fond du compteur. Le critère pratique que nous avons adopté est que l'activité minimale mesurable est celle qui donne un taux de comptage égal à trois fois le bruit de fond du détecteur.

b) Dose maximale. - Les mesures de flux instantanés diffèrent des déterminations de flux intégrés (ou des doses intégrées) quand on utilise la méthode par activation.

Dans le premier cas, le temps d'exposition n'est pas limité et même en général, pour faciliter les calculs, l'irradiation est poursuivie jusqu'à saturation de l'activité induite.

Par contre, dans le deuxième cas, si le temps d'irradiation est grand vis-à-vis de la période du produit formé, l'activité résultant n'a plus de sens par rapport à la dose reçue. En particulier si le temps d'exposition $t_{\mathrm{e}}$ est supérieur à $6 T$ (6 fois la période du produit formé) la saturation est atteinte, l'activité est constante en fonction du temps tandis que la dose reçue continue d'augmenter tant que l'exposition n'est pas arrêtée.

Cas d'impulsions neutroniques de courtes durées. Dans le cas des "flashes " de neutrons, la dose maximale mesurable n'est évidemment plus fonction de la durée d'exposition; elle dépend uniquement du temps mort du compteur.

Pratiquement on se limite à un taux de comptage maximal donnant une perte relative de comptage de l'ordre de $80 \%$; le taux de comptage limite $m_{0}$ est donc :

$$
m_{0}=0,8 / t_{\mathrm{m}}
$$

$t_{\mathrm{m}}$ étant le temps mort.

Résultats obtenus ( fig. 3 et 4). - Ainsi qu'il a été dit plus haut, les compteurs ont été soumis à des irradiations dont la durée ne dépasse pas 2T. Les résultats sont donnés après correction du temps mort et d'autoabsorption.

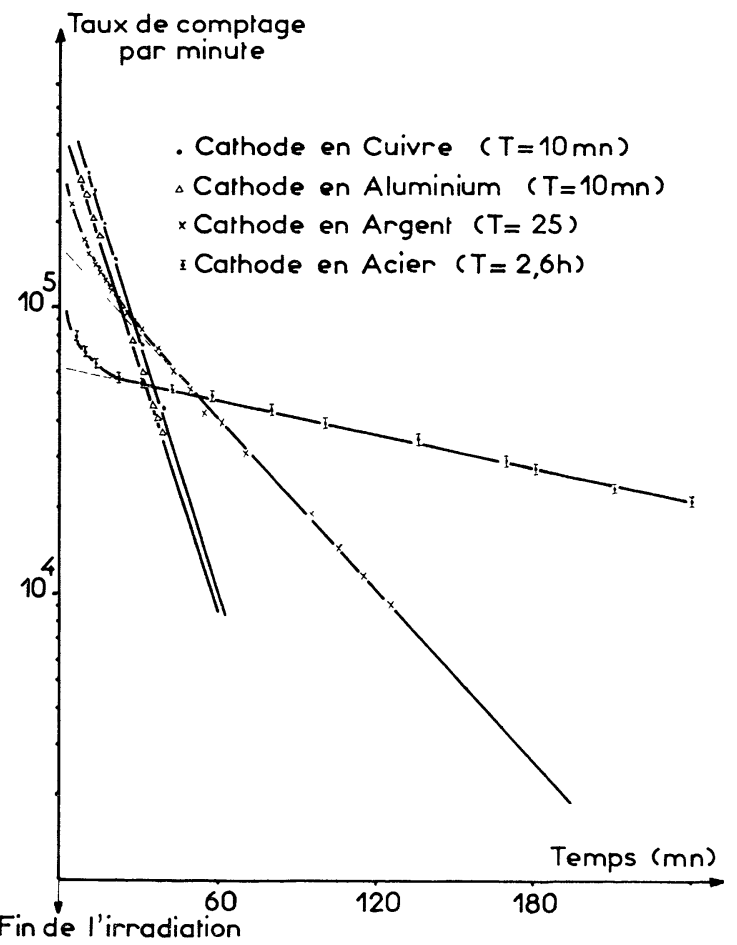

Fig. 3. - Décroissance de l'activité venant de la cathode après une irradiation totale de 5 rad.

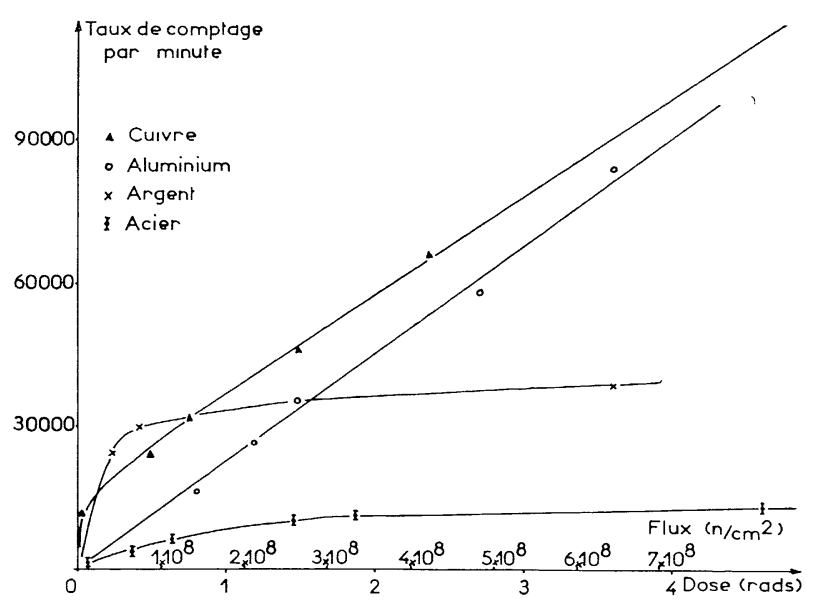

FIG. 4. - Réponses des compteurs en fonction des flux (ou doses) reçus.

Cathode d'aluminium. - La courbe de décroissance ( $f g .3$ ) montre que la seule réaction d'activation qui a lieu est ${ }_{13}^{27} \mathrm{Al}(\mathrm{n}, \mathrm{p})_{12}^{27} \mathrm{Mg}(\sigma=80 \mathrm{mb})$. Le radioélément formé a une période de $9,5 \mathrm{~min}$; le flux intégré minimal détectable est de $4 \times 10^{6} \mathrm{n} \mathrm{cm}^{-2}$ (soit une dose de 20 mrads). La 
valeur maximale mesurable est de $1,5 \times 10^{22} \mathrm{n} \mathrm{cm}^{-2}$ (dose de 8 rads environ).

Cathodes de cuivre ou de laiton. - Les résultats obtenus sont les mêmes dans les deux cas. L'activation est due à la réaction ${ }^{63} \mathrm{Cu}(\mathrm{n}, 2 \mathrm{n})^{62} \mathrm{Cu}$ $(\sigma=550 \mathrm{mb}), T=9,75 \mathrm{~m}$ et à la réaction ${ }^{63} \mathrm{Cu}(\mathrm{n}, \alpha)^{60 \mathrm{~m}} \mathrm{Co}(\sigma=23 \mathrm{mb}), T=10,5 \mathrm{~min}$.

Les courbes de décroissance mettent en évidence une période globale de 10 minutes. Le flux intégré minimal détectable est d'environ $10^{6} \mathrm{n} \mathrm{cm}^{-2}$, soit une dose de $5 \mathrm{mrad}$. Le flux maximal mesurable est de l'ordre de $1,5 \times 10^{9} \mathrm{n} \mathrm{cm}^{-2}$, soit une dose de 8 rads environ.

Cathodes D'argent. - Nous avons mis en évidence la réaction ${ }^{107} \mathrm{Ag}(\mathrm{n}, 2 \mathrm{n}){ }^{106} \mathrm{Ag}(\sigma=600 \mathrm{mb})$ donnant une période de 24 minutes. Le flux minimal mesurable est de $4 \times 10^{6} \mathrm{n} \mathrm{cm}^{-2}$ soit une dose de 20 mrads, le flux maximal est de $4 \times 10^{9} \mathrm{n} \mathrm{cm}^{-2}$ soit une dose de 20 rads.

Cathode d'acier inoxydable. - L'acier employé avait la composition suivante : Fe $68,9 \%$, Cr $17 \%$, Ni $12 \%$, Mo $<2 \%$, C $<0,1 \%$.

Seule la réaction ${ }^{56} \mathrm{Fe}(\mathrm{n}, \mathrm{p})^{56} \mathrm{Mn}(\sigma=110 \mathrm{mb})$ a été mise en évidence; le produit obtenu a une période de 2,58 heures. Les réactions possibles sur le chrome et le nickel n'ont pas été mises en évidence. Le flux minimal mesurable est de $6 \times 10^{8} \mathrm{n} \mathrm{cm}^{-2}$ soit une dose de 3 rads environ. Le flux maximal est de $7,5 \times 10^{9} \mathrm{n} \mathrm{cm}^{-2}$ soit une dose de 40 rads.

Cas d'impulsions de neutrons de très courte DURÉE. - L'activité d'un corps soumis à un flux de neutrons pendant le temps $t_{\mathrm{e}}$ est :

$$
A=N \sigma \Phi_{\mathbf{0}}\left(1-\mathrm{e}^{-\lambda t_{\mathrm{e}}}\right) .
$$

Pour un temps d'irradiation $\left(t_{\mathrm{e}}\right)$ de 0,1 seconde, nous avons calculé le flux minimal mesurable (activité égale à 3 fois le bruit de fond) à partir de l'équation (1). Le flux doit être supérieur ou égal à :

$$
\Phi_{0} \geqslant 3 B_{\mathrm{t}} / N \sigma \lambda t_{\mathrm{e}} \text {. }
$$

Pour un bruit de fond d'une impulsion par seconde, l'aluminium, le cuivre et l'argent donnent un même flux minimal égal à $10^{3} \mathrm{n} \mathrm{cm}^{-2} \mathrm{~s}^{-2}$. L'acier ne permettrait de détecter que les flux supérieurs à $10^{10} \mathrm{n} \mathrm{cm}^{-2} \mathrm{~s}^{-1}$.

On pourrait craindre que les neutrons ne détériorent les caractéristiques du détecteur, en produisant des réactions chimiques sur les molécules organiques. Pour les doses mesurées pendant les expériences (et jusqu'à $1000 \mathrm{rads}$ ) nous n'avons constaté aucune modification. Ceci a été vérifié en remplissant une cellule à analyse optique par rayons I. R., d'acétone sous une pression de 20 torrs. Après irradiation par $2 \times 10^{11}$ neutrons de $14 \mathrm{MeV}$ (dose de 1000 rads) le spectra infrarouge n'était pas modifié.

Conclusion. - Les limites de mesure de flux sont imposées par le mécanisme du fonctionnement du détecteur. La valeur minimale mesurable est fonction du bruit de fond du détecteur. La dose minimale que l'on puisse mesurer est de l'ordre de 5 mrads.

La valeur maximale détectable est limitée par le temps mort du détecteur. Dans le cas actuel, les détecteurs étant associés à des circuits de comptage classique, l'emploi d'un circuit spécial limitant le temps [11] améliorerait les performances.

Nous poursuivons nos recherches afin de mettre au point un compteur à cathode activable pour les neutrons thermiques, et un compteur permettant de faire une étude complète de l'ensemble du spectre des neutrons produits par une source de (radium- $\alpha$ beryllium).

Ces recherches ont été effectuées sous la direction du Professeur Daniel Blanc, Directeur du Centre de Physique Nucléaire de Toulouse. Nous lui exprimons notre reconnaissance pour son appui constant, et pour tous les conseils qu'ils nous a prodigués tout au long de cette étude. Ce travail a bénéficié d'un contrat de la D. R. M. E.

Manuscrit reçu le 14 janvier 1966.

\section{BIBLIOGRAPHIE}

[1] Brisbois (J.), Lott (M.) et Manent (G.), Rapport CEA-R-2491, 1964.

[2] Delattre (P.), Rapport CEA, no 1979, 1961.

[3] Eckert (R.), Rapport CEA, no 1394, 1960.

[4] Leger (P.) et Sautiez (B.), Rapport CEA, no 1267, 1959.

[5] Perriot (G.) et Schmitt (A. P.), Rapport CEA, no 2206, 1962.

[6] TARdy-Joubert (P.), Rapport CEA, nº 2250, 1963.
[7] Woenrle (M.), Rapport du C. E. N. de Grenoble, INT/Pi-171-104, 1962.

[8] Woenrle (M.), Rapport du C. E. N. de Grenoble, INT/Pi-171-115, 1962.

[9] Peirson (D. H.), J. Sc. Instr., 1956, 33, 404.

[10] Glover (R. N.), Nucl. Instr. Meth., 1958, 3, 316.

[11] Blanc (D.), Détecteurs de particules, compteurs et scintillateurs, Masson éditeur, Paris, 1959. 